\title{
Determination of Molecular Diameter by PVT
}

\author{
Vahid Moeini and Mehri Deilam \\ Department of Chemistry, Payame Noor University, P.O. Box 19395-3697 Tehran, Iran \\ Correspondence should be addressed to Vahid Moeini, v_moeini@yahoo.com
}

Received 3 December 2011; Accepted 3 January 2012

Academic Editors: S. C. Farantos and T. Panczyk

Copyright ( 2012 V. Moeini and M. Deilam. This is an open access article distributed under the Creative Commons Attribution License, which permits unrestricted use, distribution, and reproduction in any medium, provided the original work is properly cited.

\begin{abstract}
We derive an equation for calculation of molecular diameter of dense fluids, with using simultaneous Lennard-Jones (12-6) potential function and the internal pressure results. Considering the internal pressure by modeling the average configurational potential energy and then taking its derivative with respect to volume to a minimum point of potential energy has been shown that molecular diameter is function of the resultant of the forces of attraction and the forces of repulsion between the molecules in a fluid. The regularity is tested with experimental data for 10 fluids including $\mathrm{Ar}, \mathrm{N}_{2}, \mathrm{CO}, \mathrm{CO}_{2}, \mathrm{CH}_{4}, \mathrm{C}_{2} \mathrm{H}_{6}, \mathrm{C}_{3} \mathrm{H}_{8}, \mathrm{C}_{4} \mathrm{H}_{10}, \mathrm{C}_{6} \mathrm{H}_{6}, a_{\mathrm{n}}$ $\mathrm{C}_{6} \mathrm{H}_{5} \mathrm{CH}_{3}$. These problems have led us to try to establish a function for the accurate calculation of the molecular diameter based on the internal pressure theory for different fluids. The relationship appears to hold both compressed liquids and dense supercritical fluids.
\end{abstract}

\section{Introduction}

It is thus seen that all gases obey the same equation of state within the accuracy of the relation when there are no arbitrary constants specific to the individual gas. This is a statement of the principle of corresponding states. The ability of the law to predict experimental behavior is nicely shown in papers, where the reduced quantity is plotted against the reduced density at various reduced temperatures $[1,2]$.

The principle of corresponding states, introduced by J. D. van der Waals in 1880, is valid for two or more fluids with potentials of the same form but different scale parameters (say $\varepsilon$, the depth of the minimum of potential, and $r_{\min }$, the position of this minimum). This principle says that the thermodynamic properties of these fluids are equal when they are written in units of the $\varepsilon$ and $r_{\min }$ of each fluid. The potential functions of fluids satisfying the principle of corresponding states are said to be conformal [3-5].

It will have become clear that the difficulties in a statistical-mechanical treatment of pure fluids are greatest at low temperatures and high densities, that is, in the liquid region. Several models of the liquid state have been developed which provide approximate methods for calculating properties and which have some utility as bases for empirical correlations. Lattice-cell models represent one class of such theories. As the name implies, these theories are rather similar to those that might apply to the solid state, where the molecules spend most of the time at or near one location in the volume of the assemblage [6]. Despite the fact that the description of dense fluids at the molecular level is very complicated, they obey some relatively simple rules (regularities). Among these, we may refer to (1), the Taite equation that was presented by Tait about 100 years ago, (2) the linearity of the bulk modulus versus pressure for each isotherm.

A general regularity was recently reported for dense fluids, according to which $\left((\partial E / \partial V)_{T} / \rho R T\right) V^{2}$ is linear with respect to $\rho^{2}$ for each isotherm, where the quantity $(\partial E / \partial V)_{T}$ is known as the internal pressure. This equation of state works very well for all types of dense fluids, for densities greater than the Boyle density. The regularity was originally suggested on the basis of a simple lattice-type model applied to a Lennard-Jones (12-6) fluid [7, 8].

The purpose of this paper is to examine whether by the regularity, we will be able to predict of molecular diameter. The reason for expecting it to hold for fluids is the success of minimum point potential energy for fluid, in which fluids follow the same equation. In particular, we address the following points. 
(1) Initially, Lennard-Jones (12-6) potential function and the internal pressure regularity have been used to derive a equation for calculation of molecular diameter.

(2) Experimental tests of the equation for calculation of molecular diameter.

(3) If the equation holds, determine the temperature dependences of the molecular diameter. We find that the simple model that was used to predict the temperature dependences of the intercept $A$ and slope $B$ for substances can also be used to predict their temperature dependences for molecular diameter, with good agreement with experiment. The results of these calculations are also compared with other calculations which obtained using the statistical mechanical theory for calculation of molecular diameter.

\section{Theory}

Regularities, which were originally devised for normal dense fluids, are based on the cell theory and consider only nearest adjacent interaction. Regularities are applied well to Lennard-Jones fluids which their interaction potential can be modeled rather accurately by the (12-6) powers of inverse intermolecular distance. Lennard-Jones potential function suitably describes the interactions between the molecules of a fluid under the condition that it behaves as a normal fluid. These regularities were attempted to calculate the thermodynamic quantities by modeling the average configurationally potential energy and then taking its derivative with respect to volume $[9,10]$.

In the present work, using the experimental PVT, the molecular diameter will be calculated in the following stages.

Stage 1. It assumes that any kinetic energy contribution to the internal energy $E$ will vanish on taking the derivative, since the temperature is held constant. We approximate the average potential energy by summing the contribution from nearest neighbors only, assuming single inverse powers for the effective repulsion and attraction, that is,

$$
U=\frac{N}{2} z(\rho)\left(\frac{C_{i}}{\bar{r}^{i}}-\frac{C_{j}}{\bar{r}^{j}}\right),
$$

where $U$ is the total potential energy among $N$ molecules, $z(\rho)$ is the average number of nearest neighbors, $\bar{r}$ is average distance between nearest neighbors, and $C_{i}$ and $C_{j}$ are constants. It is wellknown that $z(\rho)$ to be proportional to $\rho$, as is the case for liquid argon, rubidium, cesium, and taken $v \approx$ $\bar{r}^{3}$, so that $U$ can be written as [11]

$$
\frac{U}{N}=\frac{K_{i}}{v^{(i / 3)+1}}-\frac{K_{j}}{v^{(j / 3)+1}},
$$

where $K_{i}$ and $K_{j}$ are constants. Carrying out the differentiation, we obtain the internal pressure for dense fluids.

$$
p_{\text {int }}=\left(\frac{\partial E}{\partial V}\right)_{T} \approx\left[\frac{\partial(U / N)}{\partial v}\right]_{T}=A_{1} \rho^{(i / 3)+2}-B_{1} \rho^{(j / 3)+2},
$$

where $A_{1}$ and $B_{1}$ are constants and in order to model the experimental results, it should take $j=3$ and $i=9$. Combining the foregoing results, it finds the internal pressure for dense fluids based on Lennard-Jones (12-6) potential function. It shows that the isotherm $\left((\partial E / \partial V)_{T} / \rho R T\right) V^{2}$ is linear function of $\rho^{2}$ and so predicted the temperature dependence of its parameters [8]. The final result is therefore of the form:

$$
\left(\frac{(\partial E / \partial V)_{T}}{\rho R T}\right) V^{2}=A+B \rho^{2},
$$

where the intercept $A$ and the slope $B$ significantly are related to attraction and repulsion, respectively, and both depend on temperature. In practice, it has been shown that $A=A_{1} / R T$ and $B=B_{2}-B_{1} / R T$. Thus, the intercept $A$ is proportional to $1 / T$ and the slope $B$ is functional in $1 / T$. We now consider the density problem where the system is prepared in a minimum state. Carrying out the differentiation $(\partial E / \partial V)_{T}=0$, we obtain the density for each fluid at the position of this minimum.

$$
A+B\left(\frac{\rho_{\min }}{\rho_{C}}\right)^{2}=0 .
$$

The minimum value of this density can also be written in the familiar form:

$$
\rho_{\min }=\left(\frac{-A}{B}\right)^{1 / 2} \rho_{C} .
$$

Stage 2. The Lennard-Jones potential function has been most widely used for thermodynamic and transport property prediction of substances in both pure and mixture states and in all phases of matter. However, the Lennard-Jones potential function can be written as pair-intermolecular potential to be used in thermophysical property computations, that is,

$$
U(r)=A \varepsilon\left[\left(\frac{\sigma}{r}\right)^{m}-\left(\frac{\sigma}{r}\right)^{n}\right]
$$

where $A$ is a constant, $\varepsilon$ is the potential well depths, and $\sigma$ is the diameter of molecule. The potential function obeys boundary conditions such that [12]

$$
A=\left(\frac{m}{m-n}\right)\left(\frac{m}{n}\right)^{n /(m-n)}
$$

By differentiation of the expression for the Lennard-Jones potential show that the distance $r_{\min }$ at the minimum is

$$
r_{\min }=\left(\frac{m}{n}\right)^{1 /(m-n)} \sigma,
$$

in order to model the experimental results, it should take $m=12$ and $n=6$. Where the position of this minimum of the system is given by

$$
\begin{gathered}
r_{\min }=\left(\frac{1}{\rho_{\min }}\right)^{1 / 3}, \\
\sigma=\frac{1}{2^{1 / 6}} r_{\min } .
\end{gathered}
$$


TABLE 1: Comparison between the calculated $\sigma_{P_{\text {int }}}$ and experimental values of molecular diameters $\sigma_{\eta}$ for different fluids.

\begin{tabular}{|c|c|c|c|c|c|c|c|}
\hline Fluid & $\rho / \mathrm{mol} \mathrm{L}^{-1}$ & $T / \mathrm{K}$ & $A$ & $B$ & $\Delta p / \mathrm{MPa}$ & $\sigma_{P_{\text {int }}}, \AA$ & $\sigma_{\eta}, \AA$ \\
\hline${ }^{\mathrm{a}} \mathrm{Ar}$ & 13.4074 & 200 & 0.9343 & -0.0953 & $50-600$ & 3.8249 & 3.434 \\
\hline${ }^{\mathrm{b}} \mathrm{N}_{2}$ & 11.1839 & 70 & 2.8413 & -0.2319 & $0.06-30$ & 3.9151 & 3.722 \\
\hline${ }^{\mathrm{c}} \mathrm{CO}$ & 10.7400 & 200 & 0.8460 & -0.0884 & $45-100$ & 4.1351 & 3.706 \\
\hline${ }^{\mathrm{d}} \mathrm{CO}_{2}$ & 10.6385 & 400 & 1.0995 & -0.1019 & $40-100$ & 4.0661 & 3.881 \\
\hline${ }^{\mathrm{e}} \mathrm{CH}_{4}$ & 10.0720 & 250 & 0.9904 & -0.1046 & $50-200$ & 4.2321 & 3.774 \\
\hline${ }^{\mathrm{e}} \mathrm{C}_{2} \mathrm{H}_{6}$ & 6.7600 & 400 & 1.05723 & -0.1010 & $30-70$ & 4.7535 & 4.48 \\
\hline${ }^{\mathrm{e}} \mathrm{C}_{3} \mathrm{H}_{8}$ & 4.9300 & 400 & 1.2486 & -0.0910 & $20-100$ & 5.0481 & 5.061 \\
\hline${ }^{\mathrm{e}} \mathrm{C}_{4} \mathrm{H}_{10}$ & 3.9230 & 500 & 1.1896 & -0.0824 & $30-70$ & 5.4016 & 5.339 \\
\hline${ }^{\mathrm{f}} \mathrm{C}_{6} \mathrm{H}_{6}$ & 3.8620 & 640 & 1.1561 & -0.0747 & $30-90$ & 5.3673 & 5.270 \\
\hline${ }^{\mathrm{g}} \mathrm{C}_{6} \mathrm{H}_{5} \mathrm{CH}_{3}$ & 3.1660 & 300 & 5.8742 & -0.4648 & $0.1013-100$ & 5.9318 & 932.5 \\
\hline
\end{tabular}

References ${ }^{\mathrm{a}}[20],{ }^{\mathrm{b}}[21],{ }^{\mathrm{c}}[22],{ }^{\mathrm{d}}[23],{ }^{\mathrm{e}}[24],{ }^{\mathrm{f}}[25], \mathrm{g}[26]$.

At present work, with using simultaneous of (6) of the Stage 1 and (10) of the Stage 2, the molecular diameter of each fluid can be obtained which provides a simple method to determine the molecular diameter. A typical curve of molecular diameter as a function of temperature for Ar is shown in Figure 1. This result is quite remarkable. It means that the molecular diameter of each fluid is given by the intercept $A$ and the slope $B$ significantly that are related to attraction and repulsion, respectively, and both depend on temperature. It is clear that the result is also valid for each fluid in any temperature.

\section{Internal Pressure}

Whatever the model chosen for the liquid state, the cohesive forces are of primary importance. Ignoring, for the time being, the origin of these forces, we can estimate their magnitudes from thermodynamic internal pressure. In the case of an ideal gas, the internal pressure is zero because intermolecular forces are absent. In the case of imperfect gas, the internal pressure becomes appreciable, and in the case of a liquid, it may become much greater than the external pressure [8].

The internal pressure is the resultant of the forces of attraction and the forces of repulsion between the molecules in a liquid. It therefore depends markedly on the volume $V$ and thus on the external pressure $p$. This effect is considered in the following for diethyl ether at $298 \mathrm{~K}$. For moderate increases in $p$, the internal pressure decreases only slightly, but as $p$ exceeds $500 \mathrm{MPa}$, the internal pressure begins to decrease rapidly and goes to large negative values as the liquid is further compressed. This behavior reflects on a larger scale the law of force between individual molecules; at high compressions; the repulsive forces become predominant.

Joel Hildebrand was the first to point out the significance of the internal pressure of liquids in determining solubility relationships. A solution of two liquids that differ considerably in the internal pressure usually exhibits considerable positive deviation from ideality, that is, a tendency toward lowered mutual solubility [12-17].

\section{Experimental Test}

In examining the accuracy of this work, we wish to address the following specific questions. (i) For what types of fluids is the equation valid? (ii) Over what range of density does the equations hold for these isotherms?

To see whether the regularity is limited to the certain type of fluid or is generally true, we have obtained the molecular diameter for a number of different fluids at different temperatures $[18,19]$. The various fluids tested are listed in Table 1, together with the intercept, slope, pressure range of the experimental data, and the molecular diameter for a typical isotherm. We conclude that the equation is a property of many types of dense fluids. It turns out that all the fluid isotherms hold for $\rho \geq \rho_{B} \mathrm{~mol} \mathrm{~L}^{-1}$ that this is nearly equal to the Boyle density. The Boyle temperature $T_{B}$ and Boyle volume $V_{B}$ are defined in terms of the second virial coefficient $B_{2}$ as follows: $B_{2}\left(T_{B}\right)=0$ and $V_{B}=T_{B} B_{2}^{\prime}\left(T_{B}\right)$, where $B_{2}^{\prime}=d B_{2} / d T$.

To investigate the liquid region, we turn first with fluid in its liquid range. (By liquid we mean $T \prec T_{C}$ ) Except near the critical point, all the isotherms are found to be quite linear over almost the entire density range from the vaporization line to the freezing line. The general conclusion, which is borne out for the other substances examined, is that the linear relation holds in the liquid region for $\rho \geq \rho_{B}$. In summary, we can tell, the upper density limit for the linearity in liquid region is the freezing line.

Thus, the general rule in both the liquid and supercritical regions is valid that the density holds for $\rho \geq \rho_{B}$.

\section{Discussion}

An important parameter related to the nature of fluids is the molecular diameter $\sigma$, given in terms of experimental quantities as second virial coefficient $\sigma_{B}$ and viscosity coefficient $\sigma_{\eta}$. Although authors had used several microscopic arguments in a heuristic way in obtaining universal parameters, its actual basis amounts only to the use of virial expansions for equation of state and truncation of these expansions is the 


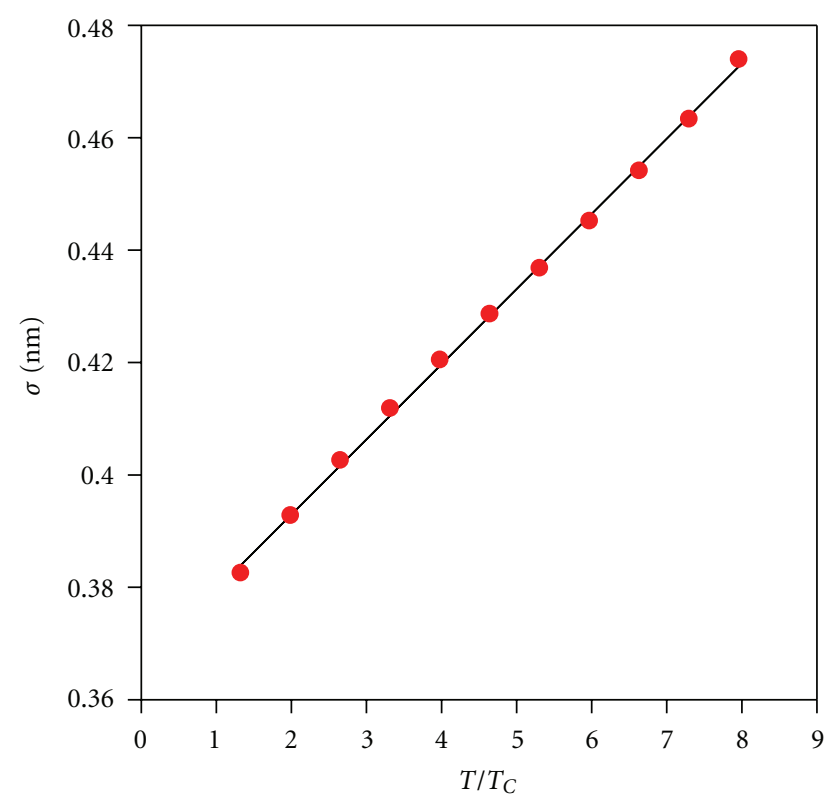

Figure 1: Typical curve of molecular diameter versus $T / T_{C}$ for $\mathrm{Ar}$ at selected.

only approximation. As justification for the accuracy of the truncation, other than good agreement with experimental data. However, there is no direct indication of any upper density limit on the molecular parameters $[15,16]$.

The molecular parameters are particularly useful in determination of molecular structure. Qualitatively, nature of molecule has been indicated by molecular diameter at given temperatures. In this paper, the internal pressure has been used to calculate molecular diameter. By use of the temperature dependence of internal pressure parameters, the molecular diameter has also been calculated at each temperature. In this paper, we have also shown that internal pressure is closely related to the molecular diameter.

The cohesive forces, which are the resultant of forces of attraction and forces of repulsion between liquid molecules, holding a liquid together create a pressure within the liquid which has been termed the internal pressure.

Internal pressure, fundamental liquid property, is closely related to the different properties of liquids such as ultrasonic velocity, free volume, viscosity, surface tension, solubility parameter, and latent heat of vaporization in the liquid phase.

The precise meaning of the internal pressure is contained in a generalized manner in the following well-known thermodynamic equation. United forces of external and internal pressure equalize the thermal pressure which tries to expand the matter. If the internal pressure of a fluid is available, then the molecular diameter can be calculated easily. The main objective of the present research is to compute the molecular diameter.

Therefore, the internal pressure was used to calculate the molecular diameter of quite different fluids for which the reliable experimental values are available. The calculated and experimental values of molecular diameter are found to present a close agreement with each other.
By careful examination of the present results, it seems to us that this work contains point of particular interest; the physical interpretation in terms of molecular forces is as follows.

If the shape of intermolecular potential function, $U(r)$, for molecules in fluids is considered, it is seen that $U(r)$ can be divided into two parts, attractive $U_{A}$ and repulsive $U_{R}$ parts, namely,

$$
U(r)=U_{A}+U_{R}
$$

Differentiation at constant temperature with respect to volume gives

$$
(\partial U(r) / \partial V)_{T}=\left(\partial U_{A} / \partial V\right)_{T}+\left(\partial U_{R} / \partial V\right)_{T},
$$

namely,

$$
P_{i}=P_{i, A}+P_{i, R},
$$

where attractive internal pressure, $P_{i, A}$ is seen to be positive that it is related to the $A$ term and repulsive internal pressure, $P_{i, R}$ is negative, therefore, it is proportional to $B$ term the internal pressure. By application of the regularity, we suggest that the molecular diameter is due to a balance between intermolecular attractive and repulsive forces [8].

A simple model had been used to predict the temperature dependences of the molecular diameter and shown to agree with experiment. At the simplest level, we now have a new and useful way of molecular diameter on the isotherms of compressed dense fluids. Moreover, the temperature dependences of molecular diameter that characterize each linear isotherm are well described by the intercept $A$ that is proportional to $1 / T$ and the slope $B$ is functional in $1 / T$.

Finally, we expect the regularity to hold for dense mixtures as well as for single substances. The reason for this 
expectation is the wellknown success of so-called one-fluid approximations for mixtures. Such approximations assume that mixtures obey the same EOS as single substances, but with parameters that depend on the composition of the mixture. Since the present linearity has been shown to be consistent with an EOS, adjusting the parameters of the EOS for compositions will not alter this result. Testing of this expectation and investigation of the composition dependences of the parameters $A$ and $B$ remain for future work [7].

\section{Acknowledgment}

The author acknowledge the Payame Noor University for the financial support.

\section{References}

[1] Y. Ghayeb, B. Najafi, V. Moeini, and G. Parsafar, "Calculation of the viscosity of supercritical fluids based on the modified Enskog theory," High Temperatures-High Pressures, vol. 35-36, no. 2, pp. 217-226, 2003.

[2] V. Moeini, F. Ashrafi, M. Karri, and H. Rahimi, "Calculation of thermal pressure coefficient of dense fluids using the linear isotherm regularity," Journal of Physics Condensed Matter, vol. 20, no. 7, Article ID 075102, 2008.

[3] H. Que and C.-C. Chen, "Photocatalytic activity of $\mathrm{TiO}_{2}$ nanoparticles sensitized by $\mathrm{CuInS}_{2}$ quantum dots," Industrial \& Engineering Chemistry Research, vol. 50, no. 19, pp. 11406$11421,2011$.

[4] F. B. Sprow and J. M. Prausnitz, "Surface tensions of simple liquid mixtures," Transactions of the Faraday Society, vol. 62, pp. 1105-1111, 1966.

[5] O. Guzmán and F. Del Río, "Theoretical equation of state of dense nonconformal fluids from effective potentials. 1: applications to model systems," Journal of Physical Chemistry B, vol. 105, no. 34, pp. 8220-8229, 2001.

[6] T. M. Reed and K. E., Gubbins, Applied Statistical Mechanics, McGraw-Hill, Rosehill, Calif, USA, 1973.

[7] A. F. Izmaylov, L. N. Shchegoleva, G. E. Scuseria, and A. Zaitsevskii, "Ab initio study of temporary anions of benzene and fluorobenzenes using the multipartitioning many-body perturbation theory," Physical Chemistry Chemical Physics, vol. 7, no. 23, pp. 3933-3937, 2005.

[8] V. Moeini, "New regularity for internal pressure of dense fluids," Journal of Physical Chemistry B, vol. 110, no. 7, pp. 3271-3275, 2006.

[9] G. Parsafar and E. A. Mason, "Linear isotherms for dense fluids: a new regularity," Journal of Physical Chemistry, vol. 97, no. 35, pp. 9048-9053, 1993.

[10] G. A. Parsafar, V. Moeini, and B. Najafi, "Pressure dependence of liquid vapor pressure: an improved gibbs prediction," Iranian Journal of Chemistry and Chemical Engineering, vol. 20, no. 1, pp. 37-43, 2001.

[11] F. Hensel and H. Uchtmann, "The metal-insulator transition in expanded fluid metals," Annual Review of Physical Chemistry, vol. 40, pp. 61-83, 1989.

[12] J. Gracia-Fadrique, "Activity coefficients at infinite dilution from surface tension data," Langmuir, vol. 18, no. 9, pp. 3604$3608,2002$.

[13] K. J. Laidler and J. H. Meiser, Physical Chemistry, The Benjamin/Cummigs Publishing Company, San Francisco, Calif, USA, 1982.
[14] W. J. Moore, Basic Physical Chemistry, Prentice/Hall International, 1983.

[15] P. W. Atkins, Physical Chemistry, Oxford University Press, 6th edition, 2001.

[16] J. O. Hirschfelder, C. F. Curtiss, and R. B. Bird, Molecular Theory of Gases and Liquids, John Wiley \& Sons, New York, NY, USA, 2nd edition, 1964.

[17] J. F. Ely and D. A. McQuarrie, "Calculation of dense fluid transport properties via equilibrium statistical mechanical perturbation theory," The Journal of Chemical Physics, vol. 60, pp. 4105-4108, 1974.

[18] C. C. Piao and M. Noguchi, "An international standard equation of state for the thermodynamic properties of HFC-125 (pentafluoroethane)," Journal of Physical and Chemical Reference Data, vol. 27, no. 4, pp. 775-806, 1998.

[19] S. L. Outcalt and M. O. McLinden, "A modified BenedictWebb-Rubin equation of state for the thermodynamic properties of R152a (1,1-difluoroethane)," Journal of Physical and Chemical Reference Data, vol. 25, no. 2, pp. 605-636, 1996.

[20] R. B. Stewart and T. Jacobsen, "Thermodynamic properties of argon from the triple point to $1200 \mathrm{~K}$ with pressures to $1000 \mathrm{MPa}$," Journal of Physical and Chemical Reference Data, vol. 18 , no. 2 , p. $639,1989$.

[21] R. T. Jacobsen, R. B. Stewart, and M. Jahangiri, “Thermodynamic properties of nitrogen from the freezing line to $2000 \mathrm{~K}$ at pressures to $1000 \mathrm{MPa}$," Journal of Physical and Chemical Reference Data, vol. 15, no. 2, pp. 735-909, 1986.

[22] R. D. Goodwin, "Carbon monoxide thermophysical properties from 68 to $1000 \mathrm{~K}$ at pressures to $100 \mathrm{MPa}$," Journal of Physical and Chemical Reference Data, vol. 14, no. 4, p. 849, 1985.

[23] R. Span and W. Wagner, "A new equation of state for carbon dioxide covering the fluid region from the triple-point temperature to $1100 \mathrm{~K}$ at pressures up to $800 \mathrm{MPa}$," Journal of Physical and Chemical Reference Data, vol. 25, no. 6, pp. 15091596, 1996.

[24] B. A. Younglove and J. F. Ely, "Thermophysical properties of fluids. II. methane, ethane, propane, isobutane, and normal butane," Journal of Physical and Chemical Reference Data, vol. 16, no. 4, p. 577, 1987.

[25] R. D. Goodwin, "Benzene thermophysical properties from 279 to $900 \mathrm{~K}$ at pressures to 1000 Bar," Journal of Physical and Chemical Reference Data, vol. 17, no. 4, p. 1541, 1988.

[26] R. D. Goodwin, "Toluene thermophysical properties from 178 to $800 \mathrm{~K}$ at pressures to 1000 Bar ," Journal of Physical and Chemical Reference Data, vol. 18, no. 4, p. 1565, 1989. 


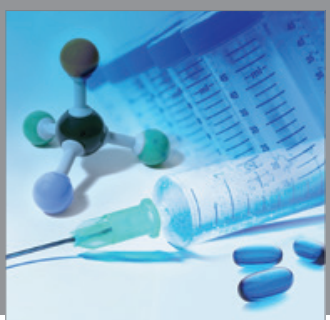

International Journal of

Medicinal Chemistry

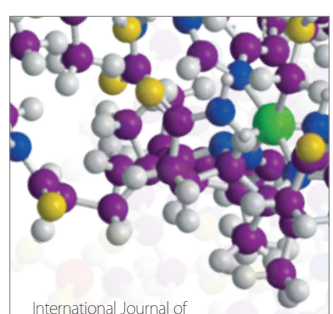

Carbohydrate Chemistry

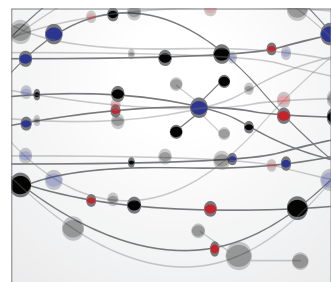

The Scientific World Journal
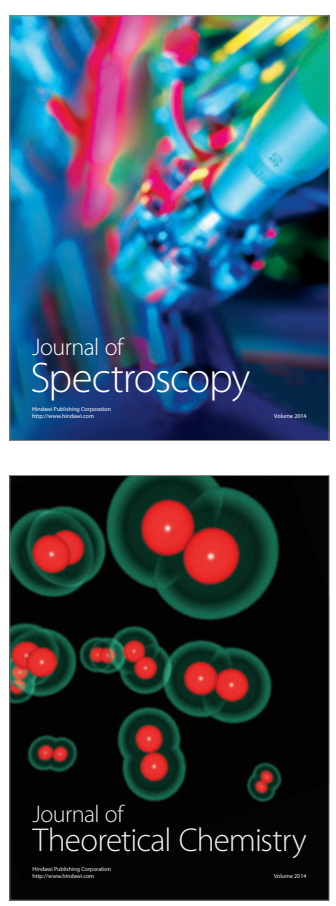
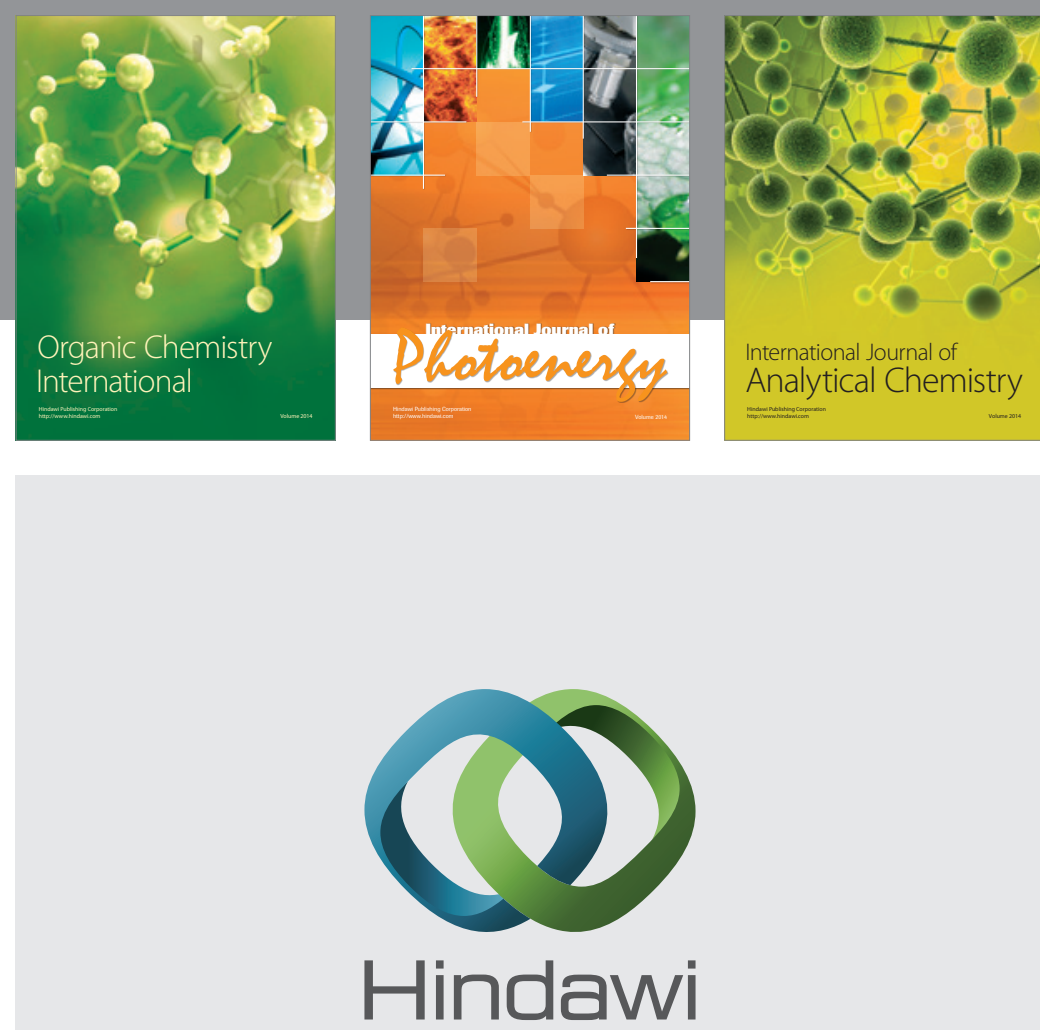

Submit your manuscripts at

http://www.hindawi.com
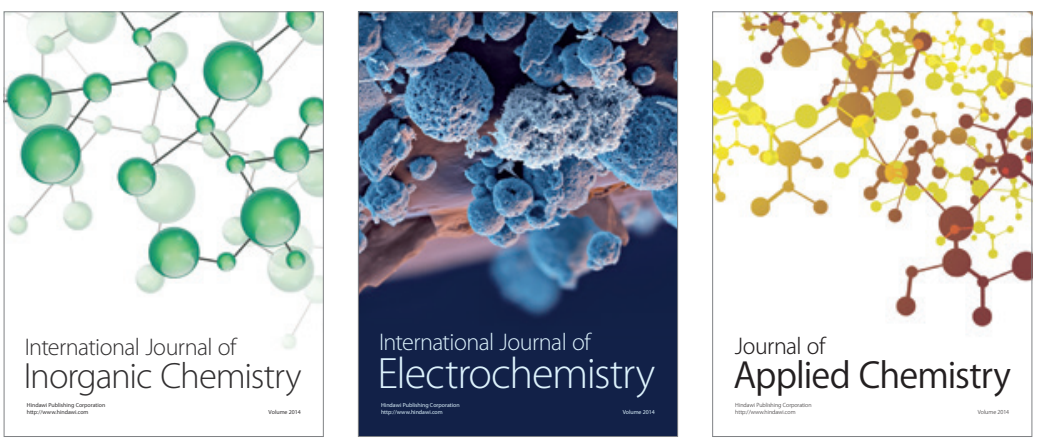

Journal of

Applied Chemistry
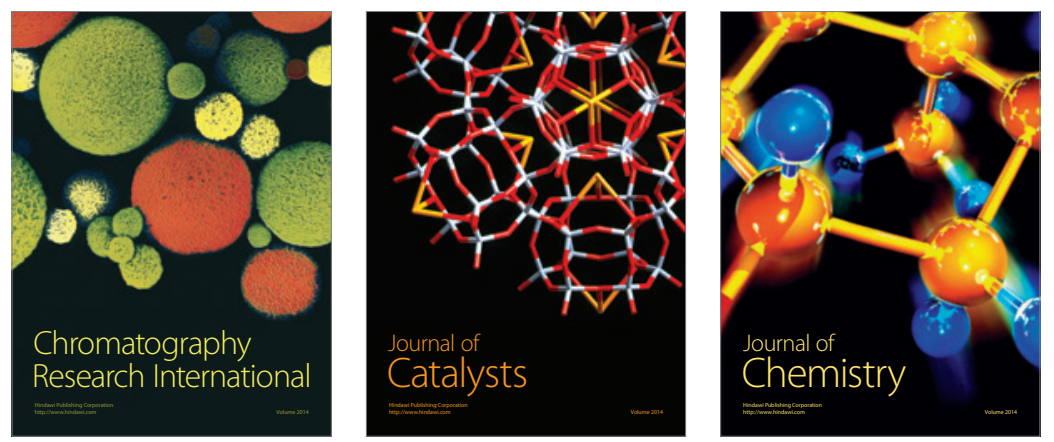
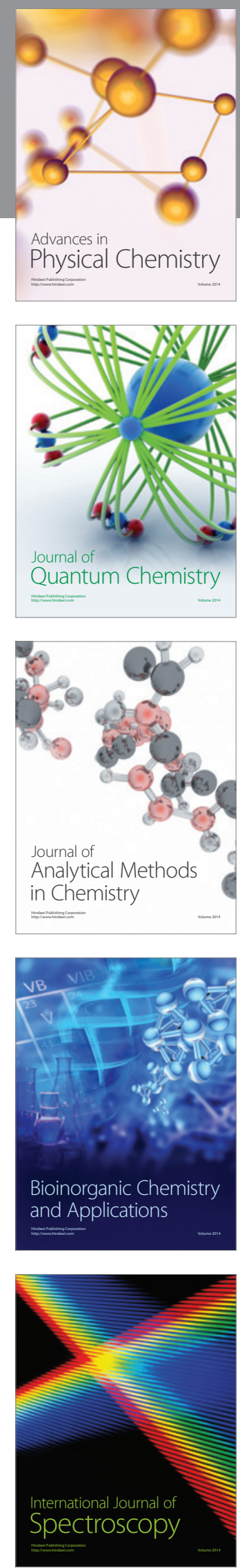